\title{
Attack Time Localization using Interval Queries
}

\author{
Nikita Ivkin \\ Amazon* \\ Gil Einziger \\ Ben Gurion University
}

\author{
Ran Ben Basat \\ Harvard University \\ Roy Friedman \\ Technion
}

\author{
Zaoxing Liu \\ Carnegie Mellon University \\ Vladimir Braverman \\ Johns Hopkins University
}

\begin{abstract}
Modern telemetry systems require advanced analytic capabilities such as drill down queries. These queries can be used to detect the beginning and end of a network anomaly by efficiently refining the search space. We present the first integral solution that $(i)$ enables multiple measurement tasks inside the same data structure, (ii) supports specifying the time frame of interest as part of its queries, and (iii) is sketch-based and thus space efficient. Namely, our approach allows the user to define both the measurement task (e.g., heavy hitters, entropy estimation, cardinality estimation) and the time frame of relevance (e.g., 5PM-6PM) at query time. Our approach provides accuracy guarantees and is the only space-efficient solution that offers such capabilities. Finally, we demonstrate how the algorithm can be used to accurately pinpoint the beginning of a realistic DDoS attack.
\end{abstract}

\section{CCS CONCEPTS}

- Networks $\rightarrow$ Data path algorithms; Network algorithms;

ACM Reference Format:

Nikita Ivkin, Ran Ben Basat, Zaoxing Liu, Gil Einziger, Roy Friedman, and Vladimir Braverman . 2019. Attack Time Localization using Interval Queries. In SIGCOMM '19: ACM SIGCOMM 2019 Conference (SIGCOMM Posters and Demos '19), August 19-23, 2019, Beijing, China. ACM, New York, NY, USA, 3 pages. https://doi.org/10.1145/3342280.3342316

\section{INTRODUCTION}

Network monitoring is at the heart of many networking protocols and network functions, such as traffic engineering [8], attack and anomaly detection [7], and forensic analysis [13]. Over the years, a large number of traffic metrics have been defined, such as heavy hitter detection [3, 5, 6], cardinality estimation [12], and entropy estimation [2]. Given limited memory and computing resources on the network devices, network telemetry systems usually provide approximate results with sufficient accuracy guarantee.

However, existing algorithms are usually designed for estimating the metrics in a predefined, fixed measurement window without the ability to zoom into any sub-region of the window. This lack of

\footnotetext{
*This work was done while the author was at Johns Hopkins University.

Permission to make digital or hard copies of all or part of this work for personal or classroom use is granted without fee provided that copies are not made or distributed for profit or commercial advantage and that copies bear this notice and the full citation on the first page. Copyrights for components of this work owned by others than ACM must be honored. Abstracting with credit is permitted. To copy otherwise, or republish, to post on servers or to redistribute to lists, requires prior specific permission and/or a fee. Request permissions from permissions@acm.org.

SIGCOMM Posters and Demos '19, August 19-23, 2019, Beijing, China

(c) 2019 Association for Computing Machinery.

ACM ISBN 978-1-4503-6886-5/19/08 . \$ \$15.00

https://doi.org/10.1145/3342280.3342316
}
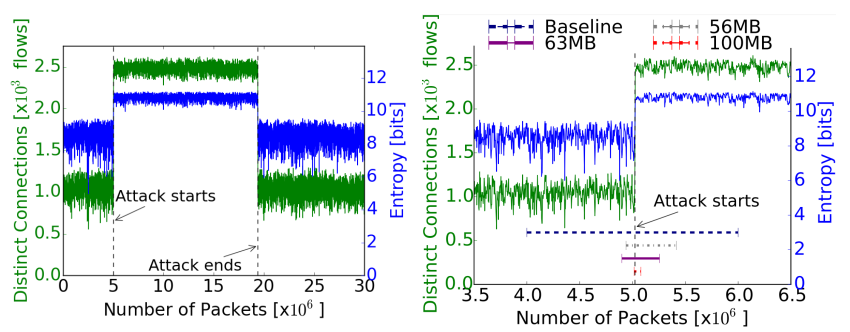

Figure 1: The impact of a DDoS attack on the Distinct Count (Green), and the Entropy (Blue) metrics. On the right, the result of our attack pinpointing algorithm for given space allocations.

visibility inside the window can potentially fail to capture important events that we want to monitor.

For instance, an attack detector may wish to identify the beginning and the end of a DDoS attack. To accurately detect such an event, as illustrated in Figure 1, we need efficient algorithms to monitor significant changes in the entropy or number of distinct flows in some small intervals of the measurement window.

In this context, the aforementioned attack monitoring problem can be solved in the Interval Query (IQ) $[4,18]$ model, which generalizes sliding window to provide visibility to any interval inside the window. In the IQ model, the user provides the interval of reference at query time, and the metric is estimated over that interval. It is suitable to accurately pinpoint the beginning of an attack (as in Figure 1) or for identifying short and important patterns. For example, recent work $[4,18]$ proposes efficient solutions to track $L_{1}$-Heavy Hitters in the IQ model.

In this poster, we present a measurement framework that supports queries with $L_{2}$-guarantee in the IQ model. Pushing the limit to $L_{2}$ allows us to estimate important metrics which are useful in attack detection including $L_{2}$-heavy hitters, distinct flows, and entropy [11, 17]. In addition, it enables us to extend general sketches (e.g., UnivMon [16]) to the IQ model. To the best of our knowledge, our work is the first to study the IQ model with $L_{2}$ guarantee.

\subsection{Interval Queries with $L_{2}$-Guarantee}

We seek to identify approximate $L_{2}$ heavy hitter flows in the IQ model. That is, at time $t$, the user issues a query $\left(t_{1}, t_{2}\right) \subset(t-n, t)$ that returns all flows whose packets appear in the interval $\left(t_{1}, t_{2}\right)$ at least $\varepsilon \cdot L_{2}\left(t_{1}, t\right)$, and no flow with frequency less than $\frac{\varepsilon}{2} L_{2}\left(t_{1}, t\right)$. The notation $L_{2}\left(t_{1}, t\right)$ is the $L_{2}$ norm of the interval $\left(t_{1}, t\right)$.

To design the algorithm, we use Smooth Histogram (SH) [10] technique and adapt state-of-the-art $L_{2}$-heavy hitters sketches [ 9 , 15]. To be concise, we only present the high-level intuition and bounds.

By definition, any flow that is heavy on the interval $\left(t_{1}, t_{2}\right)$ is also heavy on the suffix $\left(t_{1}, t\right)$; however, the reverse logic does not follow. 


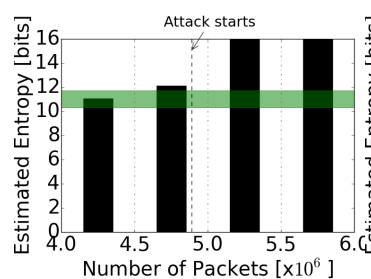

(a) Step 0

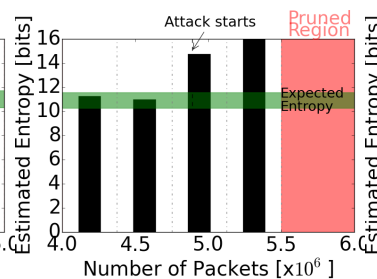

(b) Step 1

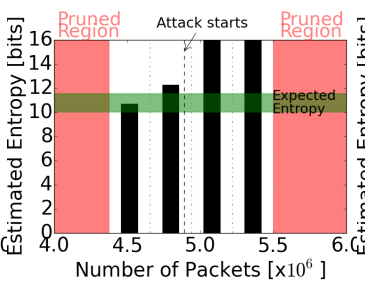

(c) Step 2

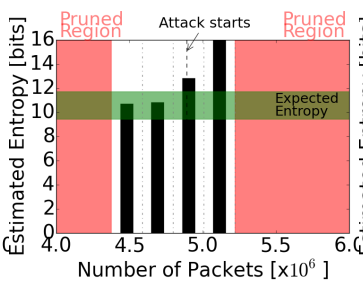

(d) Step 3

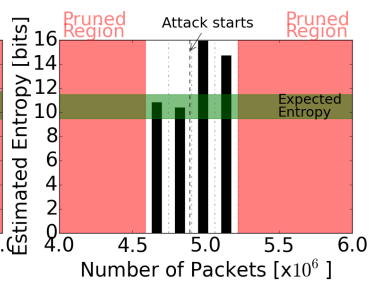

(e) Step 4

Figure 2: An illustration of the first steps of the attack pinpointing algorithm (with $63 \mathrm{MB}$ ). In steps 1, 3, and 4, the first unexpected entropy appeared in the last two sub-intervals, allowing the algorithm to prune the first sub-interval. In steps 0 and 2, the algorithm already encounters abnormally high entropy in the second sub-interval and therefore prunes the latest sub-interval. For reference, we annotate the actual starting time of the attack (which is not known to the algorithm).

We show that sliding window frameworks (such as $\mathrm{SH}$ ) can be used to identify heavy hitters on any prefix $\left(t_{1}, t\right)$, and we use it as a set of potentially heavy flows on any interval $\left(t_{1}, t_{2}\right)$. For each potentially heavy flow, we run an IQ counting algorithm, in parallel we run IQ $L_{2}$ norm estimator for the entire stream. The approximate candidate count and $L_{2}$ norm on a given interval let us verify if it is heavy on the interval. Our algorithm requires $O\left(\varepsilon^{-3} \log ^{2} n\right)$ memory, and works with $O(\log n)$ update time and $O\left(\varepsilon^{-2} \log n\right)$ query time.

$L_{2}$-guarantee in IQ model paves the route to interval Univmon Sketch [16]. We theoretically extend Univmon to the IQ model and use our $L_{2}$ algorithm as a building block towards maintaining a variety of metrics: entropy, cardinality, and others, with only one sketch.

\subsection{Attack localization algorithm}

We now explore the attack localization capability, which is facilitated by $L_{2}$ heavy hitter algorithms in the IQ model. We use the entropy signal, which is useful for detecting a variety of attacks $[14,17]$. In addition to attack detection, we also attempt to estimate when the attack started. Our attack localization algorithm periodically estimates the entropy within a sliding window, e.g., once per $1 \mathrm{M}$ packets, and maintains the average and standard deviation of the entropy over previous windows. We identify a possible attack when the current entropy estimation is not within five standard deviations from the past entropy. At that point, we begin the attack localization process. The delicate point to consider is that the attack did not necessarily start within the window that raised the alarm. It is possible that the attack began within the previous window but did not build enough impact to trigger the alarm. Therefore, our Baseline interval for the start of the attack is the last two windows. Note that any sliding window entropy algorithm (e.g., [2]) reaches the Baseline localization.

Next, we attempt to narrow the suspected interval recursively. We partition the current interval (e.g., 4M-6M packets) into four equal parts (e.g., [4M,4.5M], [4.5M,5M], [5M,5.5M], and [5.5M,6M]) and estimate the entropy on each of them. We note that entropy is not linear, and we cannot compare the entropy of a short interval to that of a long interval. Instead, we use the entropy of equal sized subintervals that happened before Baseline to define the 'normal' entropy. That is, we calculate the mean and standard deviation of such intervals. We call these safe intervals as they are before the attack. In this example, we estimate the entropy of [3.5M,4M], $[3 \mathrm{M}, 3.5 \mathrm{M}]$, etc. We favor the most recent safe intervals as the normal

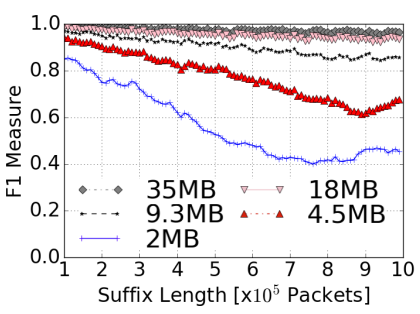

(a) Heavy Hitters

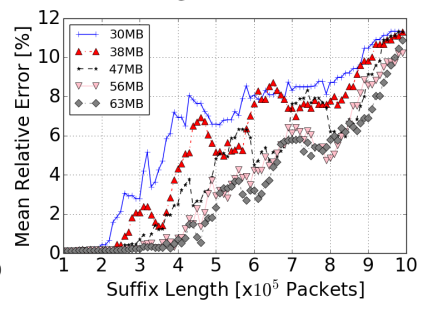

(b) Entropy Estimation
Figure 3: Evaluation for (a) $L_{2}$-Heavy Hitters and (b) Distribution Entropy Estimation.

behavior may gradually change, and for algorithmic considerations as the estimation error for these intervals is smaller (see Figure 3).

We set the new suspected interval as a concatenation of all abnormal intervals, and recursively repeat the above process until we can no longer shorten the suspected interval. The operation of the algorithm is illustrated in Figure 2. Specifically, if one of the first two subintervals had unexpectedly high entropy (as in steps 0 and 2), we prune the latest subinterval. Otherwise (as in steps 1,3, and 4), we can safely prune the first subinterval as we expect the entropy to increase after at least one complete attack subinterval.

\section{EVALUATION}

We implemented a prototype in $\mathrm{C}$ and evaluated the accuracy for various measurements using the CAIDA Internet Trace NY2018 [1].

Entropy and Heavy Hitters: We evaluate the errors in Entropy estimation and $L_{2}$-Heavy Hitters. As illustrated in Figure 3, our solution improves with more space and is more accurate on recent intervals.

DDoS attack generation: We simulate an attack containing numerous attackers from 50 randomly picked subnets and multiple victims sharing a single 16-bit subnet, similar to the one considered in [3]. The high number of attackers and the low per-attacker traffic makes attack localization impossible using existing IQ techniques.

Localization results: Figure 1 depicts the actual entropy and distinct count of the stream and the attack start time. In addition, it presents the localization output for different memory allocations. Our results show that (i) the Baseline solution using sliding windows is inaccurate for the task; that is, while existing methods may identify the attack they cannot localize it. (ii) Our attack localization successfully localizes the beginning of the attack; and (iii) there is a trade-off between the localization's accuracy and the allocated memory. More memory allows for better localization. 


\section{REFERENCES}

[1] The CAIDA nonymized Internet Traces equinix-nyc 2018-03-15, Dir. A.

[2] Assaf, E., Ben-BAsat, R., EinZiger, G., and Friedman, R. Pay for a sliding bloom filter and get counting, distinct elements, and entropy for free. In INFOCOM 2018

[3] Basat, R. B., Einziger, G., Keslassy, I., Orda, A., Vargaftik, S., and Waisbard, E. Memento: Making sliding windows efficient for heavy hitters. In CoNEXT 2018

[4] BASAT, R. B., Friedman, R., AND Shahout, R. Heavy hitters over interval queries. In PVLDB (2019). Also available on arXiv:1804.10740.

[5] Ben-Basat, R., Einziger, G., Friedman, R., and Kassner, Y. Heavy hitters in streams and sliding windows. In IEEE INFOCOM (2016).

[6] Ben-Basat, R., Einziger, G., Friedman, R., And Kassner, Y. Randomized admission policy for efficient top-k and frequency estimation. In INFOCOM (2017).

[7] Ben-Basat, R., Einziger, G., Friedman, R., Luizelli, M., and Waisbard, E. Constant time updates in hierarchical heavy hitters. In ACM SIGCOMM (2017).

[8] Benson, T., Anand, A., Akella, A., And Zhang, M. MicroTe: Fine Grained Traffic Engineering for Data Centers. In ACM CoNEXT (2011)

[9] Braverman, V., Chestnut, S. R., Ivkin, N., Nelson, J., Wang, Z., and WoodrufF, D. P. Bptree: an $\ell_{2}$ heavy hitters algorithm using constant memory. arXiv:1603.00759.

[10] Braverman, V., AND Ostrovsky, R. Smooth histograms for sliding windows. In
IEEE FOCS (2007).

[11] Feibish, S. L., Afek, Y., Bremler-Barr, A., Cohen, E., and Shagam, M. Mitigating DNS random subdomain ddos attacks by distinct heavy hitters sketches. In ACM/IEEE HotWeb 2017.

[12] Heule, S., Nunkesser, M., ANd Hall, A. Hyperloglog in practice: Algorithmic engineering of a state of the art cardinality estimation algorithm. In EDBT (2013).

[13] Kaushik, A. K., Pilli, E. S., AND Joshi, R. C. Network forensic analysis by correlation of attacks with network attributes. In Inf. and Comm. Tech. (2010).

[14] Kumar, K., Joshi, R., AND Singh, K. A distributed approach using entropy to detect ddos attacks in isp domain. In IEEE ICSCN (2007).

[15] Liu, Z., Ben-Basat, R., Einziger, G., Kassner, Y., Braverman, V., Friedman, R., AND SEKAR, V. Nitrosketch: Robust and general sketch-based monitoring in software switches. In ACM SIGCOMM (2019).

[16] Liu, Z., Manousis, A., Vorsanger, G., Sekar, V., and Braverman, V. One sketch to rule them all: Rethinking network flow monitoring with univmon. In $A C M$ SIGCOMM (2016).

[17] Nychis, G., Sekar, V., Andersen, D. G., Kim, H., and Zhang, H. An empirical evaluation of entropy-based traffic anomaly detection. In IMC (2008).

[18] Papapetrou, O., Garofalakis, M., and Deligiannakis, A. Sketching distributed sliding-window data streams. VLDB fournal (2015). 